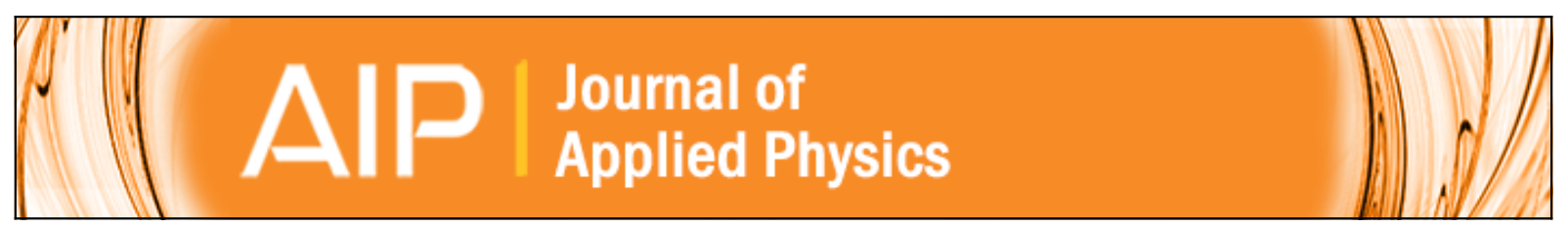

\title{
Importance of ion bombardment during coverage of Au nanoparticles on their structural features and optical response
}

V. Resta, R. J. Peláez, and C. N. Afonso

Citation: Journal of Applied Physics 115, 124303 (2014); doi: 10.1063/1.4869559

View online: http://dx.doi.org/10.1063/1.4869559

View Table of Contents: http://scitation.aip.org/content/aip/journal/jap/115/12?ver=pdfcov

Published by the AIP Publishing

\section{Articles you may be interested in}

Growth of Au nanoparticle films and the effect of nanoparticle shape on plasmon peak wavelength

J. Appl. Phys. 115, 193506 (2014); 10.1063/1.4876263

Electrical and optical properties of vanadium dioxide containing gold nanoparticles deposited by pulsed laser deposition

Appl. Phys. Lett. 101, 133102 (2012); 10.1063/1.4754708

Au-nanoparticles-decorated MWCNTs demonstrating enhanced fluorescence and Raman spectroscopy AIP Conf. Proc. 1451, 58 (2012); 10.1063/1.4732367

In-situ and ex-situ characterization of $\mathrm{TiO} 2$ and $\mathrm{Au}$ nanoparticle incorporated $\mathrm{TiO} 2$ thin films for optical gas sensing at extreme temperatures

J. Appl. Phys. 111, 064320 (2012); 10.1063/1.3695380

Coverage induced regulation of Au nanoparticles during pulsed laser deposition

J. Appl. Phys. 109, 094302 (2011); 10.1063/1.3549168

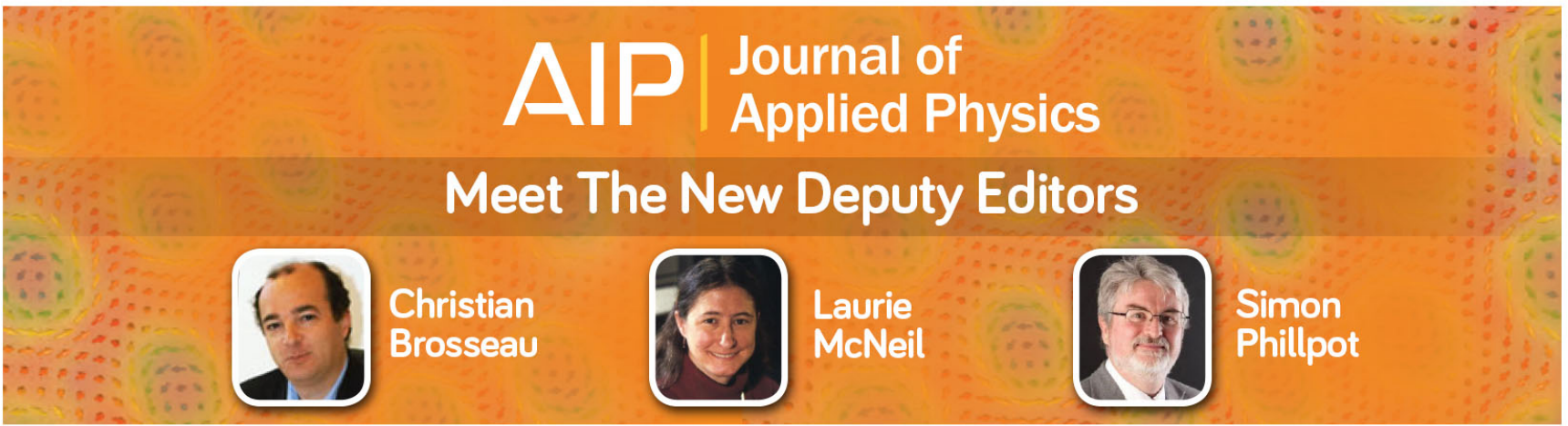




\title{
Importance of ion bombardment during coverage of Au nanoparticles on their structural features and optical response
}

\author{
V. Resta, ${ }^{a}{ }^{2}$ R. J. Peláez, and C. N. Afonso \\ Laser Processing Group, Instituto de Óptica, CSIC, Serrano 121, E-28006 Madrid, Spain
}

(Received 11 February 2014; accepted 14 March 2014; published online 25 March 2014)

\begin{abstract}
This work studies the changes in the optical response and morphological features of $6 \pm 1 \mathrm{~nm}$ diameter $\mathrm{Au}$ nanoparticles (NPs) when covered by a layer of a- $\mathrm{Al}_{2} \mathrm{O}_{3}$ by pulsed laser deposition (PLD). The laser fluence used for ablating the $\mathrm{Al}_{2} \mathrm{O}_{3}$ target is varied in order to modify the kinetic energy (KE) of the species bombarding the NPs during their coverage. When the ion $\mathrm{KE}<200 \mathrm{eV}$, the structural features and optical properties of the NPs are close to those of uncovered ones. Otherwise, a shift to the blue and a strong damping of the surface plasmon resonance is observed as fluence is increased. There are two processes responsible for these changes, both related to aluminum ions arriving to the substrate during the coverage process, i.e., sputtering of the metal and implantation of aluminum species in the metal. Both processes have been simulated using standard models for ion bombardment, the calculated effective implanted depths allow explaining the observed changes in the optical response, and the use of a size-dependent sputtering coefficient for the Au NPs predicts the experimental sputtering fractions. In spite of the work is based on PLD, the concepts investigated and conclusions can straightforwardly be extrapolated to other physical vapor deposition techniques or processes involving ion bombardment of metal NPs by ions having KE > 200 eV. (C) 2014 AIP Publishing LLC. [http://dx.doi.org/10.1063/1.4869559]
\end{abstract}

\section{INTRODUCTION}

The unique optical properties of noble metal nanoparticles (NPs) arise from an absorption band related to the surface plasmon resonance (SPR) whose position depends on the dimensions, shape, and environment. ${ }^{1-3}$ For many applications, the NPs should be embedded in a solid medium whose main effect is a slight shift of the SPR to the red as the host refractive index increases. ${ }^{4,5}$ Indeed, several results evidence the different features of the SPR depending on whether the NPs are dissolved in a liquid, at the surface of a substrate or embedded in a solid medium. However, these variations are mainly discussed in terms of the different environment/arrangement and are very scarcely related to the production procedure. ${ }^{5-7}$

The most versatile approach to produce embedded NPs in layers is the use of thin film technologies for producing in-situ both the metal NPs and the embedding/covering layer. Sputtering and pulsed laser deposition (PLD) have successfully been used to produce such embedded metal NPs in an oxide host. $^{5-7}$ For the case of Au NPs embedded in an a- $\mathrm{Al}_{2} \mathrm{O}_{3}$ host produced by PLD, a competition between surface growth and sputtering effects has been reported leading to a regulation of the dimensions of the NPs at high fluences. ${ }^{8}$ While the impact of the covering process on the morphology of NPs has been documented to some extent, there are much less reports on the impact on their optical response. Evidences for coverage induced reactive processes that change the plasmonic response of $\mathrm{Cu}$ and $\mathrm{Ag}$ NPs have been

\footnotetext{
${ }^{a)}$ Present address: CEDAD - CEnter for DAting and Diagnostics, Department of Engineering for Innovation, University of Salento, c/o Cittadella della Ricerca SS. 7 Km. 706, I-72100 Brindisi, Italy.
}

reported, ${ }^{5,6}$ as well as the effect of covering Au NPs as a function of the initial NPs dimensions. ${ }^{7}$ A significant shift to the blue and damping of the SPR was observed and related to the sputtering of the metal by species of the covering layer arriving with kinetic energies (KE) higher than $200 \mathrm{eV}$, the smaller the NPs the higher the sputtering fraction.

Ion bombardment of solid surfaces is known since very long to result in several surface modifications, the more widely studied ones being sputtering of atoms from the surface, implantation of species under the surface and surface damage by creating defects and/or erosion. ${ }^{9}$ It has more recently received lot of attention as a means to produce metal NPs from a thin metal layer ${ }^{10-14}$ or modify arrays of NPs. ${ }^{15}$ In the former case, almost spherical NPs are produced and a bimodal distribution of large and small NPs is generally observed. While there is a general agreement on the large ones are related to ion induced dewetting, the small ones are discussed in terms of re-deposition ${ }^{13}$ or produced under the surface by implantation in the substrate. ${ }^{11,12,14}$ The latter explanation is the most likely when comparing these bimodal distributions imaged in plan-view to those reported for covered metal NPs produced by PLD and for which cross-section images clearly demonstrated the small NPs were inside the substrate. $^{8}$ In addition, sputtering of metal was generally observed and the experimentally determined sputtering yield (SY) was simulated using standard models that lead to values that were smaller than the experimental ones ${ }^{7,10,15}$ and was found to increase with dose. ${ }^{12}$ However, in spite of there are evidences that the SY depends on the size of the nanostructures, ${ }^{7,15}$ its detailed dependence is still an open question.

The aim of this work is to study the effects caused during the coverage process by ion bombardment as a function of the ion KE while keeping constant the initial morphology 
of the NPs. The KE of the species is varied through the laser fluence used to ablate the $\mathrm{Al}_{2} \mathrm{O}_{3}$ target in a range such that the percentage of ions with $\mathrm{KE}>200 \mathrm{eV}$ varies from being negligible up to a value as high as $75 \% .{ }^{16}$ Ultimately, the work aims to determine whether the laser fluence can be a means to suppress or reduce the effects of ion bombardment during growth. The most widely used alternative way to reduce ion bombardment during PLD is introducing a gas pressure that in addition to negative aspects related to gas phase collisions and reactions, it produces a host with a porous surface and an increased density of defects that prevents the PLD nanostructuring capabilities. ${ }^{17}$ In spite of the work is performed using PLD, the concepts here investigated and conclusions can straightforwardly be extrapolated to other physical vapor deposition techniques or processes involving ion bombardment.

\section{EXPERIMENT}

The samples have been produced by alternate PLD in vacuum $\left(5 \times 10^{-6}\right.$ millibars $)$ by focusing an $\mathrm{ArF}$ laser beam $(\lambda=193 \mathrm{~nm}, \tau=20 \mathrm{~ns}$ full width half maximum (FWHM)) on bulk ceramic $\mathrm{Al}_{2} \mathrm{O}_{3}$ and $\mathrm{Au}$ targets at an angle of $45^{\circ}$ with respect to its normal. The laser repetition rate has been set at $20 \mathrm{~Hz}$. The substrate was held at room temperature and placed $40 \mathrm{~mm}$ away from the target. Deposition was performed simultaneously on three types of substrates, each having a part within the central homogeneous area: a $1 \mathrm{~mm}$ thick glass plate, a [100] $\mathrm{Si}$ wafer, and a carbon-coated mica substrate. A fixed fluence of $\mathrm{F}_{0}=2.7 \mathrm{~J} \mathrm{~cm}^{-2}$ has been used to produce both the NPs and a $10 \mathrm{~nm}$ thick a- $\mathrm{Al}_{2} \mathrm{O}_{3}$ buffer layer before the ablation of the gold target in order to provide the same nucleation surface for the NPs regardless the substrate used. The a- $\mathrm{Al}_{2} \mathrm{O}_{3}$ covering layer has instead been produced by ablating the $\mathrm{Al}_{2} \mathrm{O}_{3}$ target with different $\mathrm{X} \mathrm{F}_{0}$ fluences with $\mathrm{X}$ in the range of 0.3-3.3. In order to determine the number of pulses required to achieve the desired layer thickness or amount of metal, the reflectivity of calibration samples was in situ measured as reported elsewhere. ${ }^{8}$ The number of pulses for ablating the gold target was fixed to 640, while the number of pulses to ablate the $\mathrm{Al}_{2} \mathrm{O}_{3}$ target during the coverage was varied in the range 980 to 17380 in order to produce an approximately $10 \mathrm{~nm}$ thick covering layer in all cases, the higher the fluence the lower the number of pulses. Finally, a sample with no covering layer was produced that will be referred to from now on as reference sample. Further details on the deposition procedure can be found elsewhere. ${ }^{7,8}$

The optical extinction spectra of the specimens produced on glass substrates have been calculated as $\ln (1 / T)$, where $T$ is the transmission measured in the range $400-800 \mathrm{~nm}$ using a spectroscopic ellipsometer (WVase) at normal incidence. The gold content has been determined in the specimens produced on Si substrates, by Rutherford Backscattering (RBS) using a $3 \mathrm{MeV}$ proton beam. The morphology of the Au NPs has been characterized by transmission electron microscopy (TEM). Specimens for plan view observations have been prepared by floating off the films from the carbon-coated mica substrate in de-ionized water and picking them up on copper grids for observation. TEM measurements have been performed using TECNAI F30 TEM operating at $300 \mathrm{kV}$ and point-to-point resolution of $0.205 \mathrm{~nm}$. The image analysis has been performed by studying areas of at least $200 \times 200 \mathrm{~nm}^{2}$ by means of the ImageJ software. ${ }^{18}$ The NPs diameter is finally determined by averaging the measured length $l$ (longer in-plane dimension) and width $w$ (in-plane dimension perpendicular to $l$ ) in the binary images.

\section{RESULTS}

Figure 1 shows the extinction spectra of samples produced with increasing fluences to ablate the $\mathrm{Al}_{2} \mathrm{O}_{3}$ target, together with the spectrum of the reference sample. All spectra show a broad band related to the SPR whose maximum will be referred to from now on as the SPR wavelength. It is worth pointing out that the reference sample is equivalent to the on sample produced with $\mathrm{F}_{0}$ and having a metal content of $11 \times 10^{15}$ atoms cm $^{-2}$ reported earlier. ${ }^{7}$ For the lowest fluence used, the spectral features are very similar to those of the reference sample, the only significant difference being its higher absorption. As fluence increases, the extinction decreases, and the SPR wavelength shifts to the blue. For fluences $>F_{0}$, the SPR is no longer noticeable and a monotonous decrease of the intensity from UV towards the IR is instead observed.

Figure 2 shows plan view images of the reference sample (a) and samples produced with increasing fluences to ablate the $\mathrm{Al}_{2} \mathrm{O}_{3}$ target for the covering layer. All images show a bimodal distribution of metal NPs (dark areas) that is related to implantation inside the buffer a- $\mathrm{Al}_{2} \mathrm{O}_{3}$ layer that acts as substrate (small NPs with diameter $<1.7 \mathrm{~nm}$ ) and to NPs that are formed at its surface (bigger ones). ${ }^{8,19}$ From now on, we will neglect the former NPs and refer to the latter ones as NPs. Before covering, these NPs have an average diameter of $6 \pm 1 \mathrm{~nm}$ and cover $\approx 30 \%$ of the surface. After coverage using the highest fluence studied, the diameter and coverage of NPs reduce down to $3 \pm 1 \mathrm{~nm}$ and $7 \%$, respectively.

Since in earlier works, it was shown that the amount of gold implanted atoms or dose saturates around $1.7 \times 10^{15}$ atoms $\mathrm{cm}^{-2}, 7,8$ the metal content of the NPs at the surface $[\mathrm{Au}]$ is thus calculated by detracting this dose to the amount of number of gold atoms determined experimentally by RBS. Figure 3(a) shows [Au], the mean diameter of the NPs

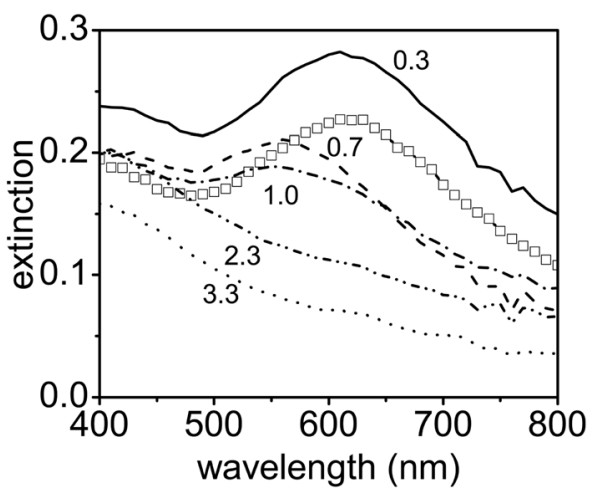

FIG. 1. Extinction spectra of samples produced at increasing $\mathrm{X} \mathrm{F}_{0}$ fluences to ablate the $\mathrm{Al}_{2} \mathrm{O}_{3}$ target during the production of the covering layer, the labels of the curves correspond to $\mathrm{X}$ and $\mathrm{F}_{0}=2.7 \mathrm{~J} \mathrm{~cm}^{-2}$. The spectrum of the reference sample $(\square)$ is also included. 

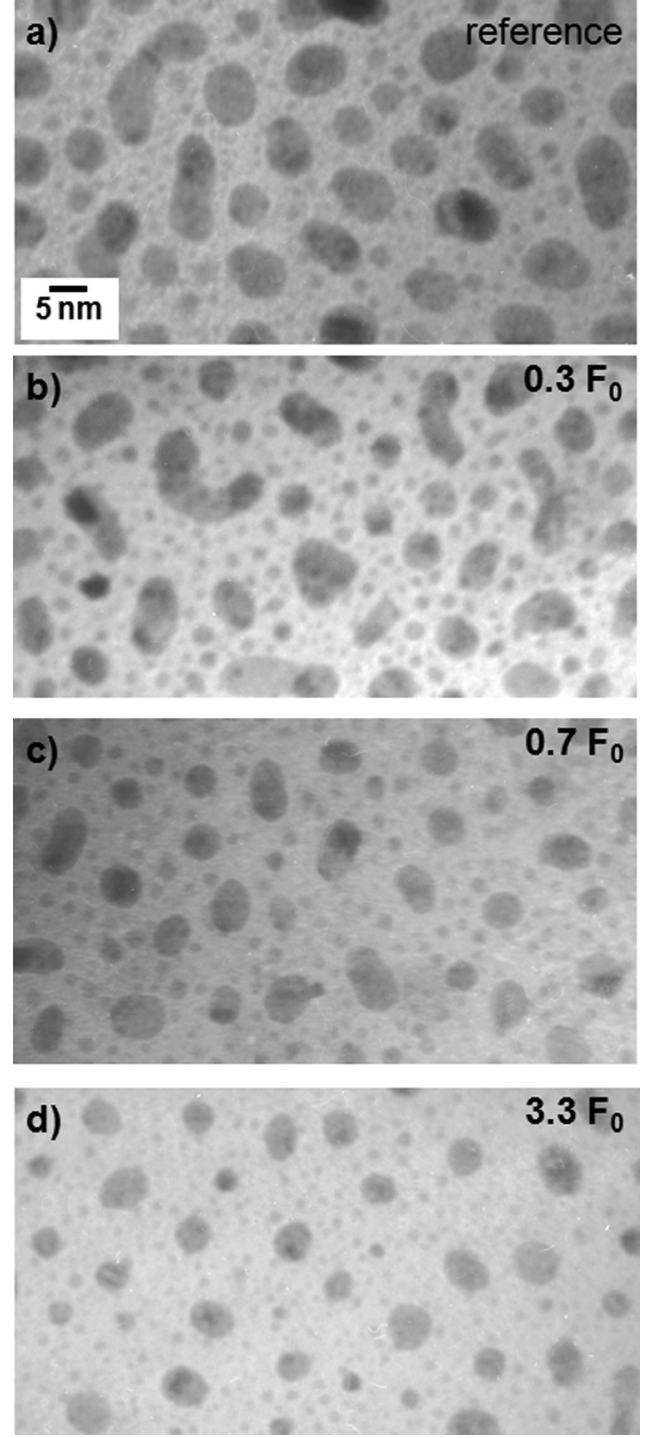

FIG. 2. TEM images of the (a) reference sample and (b)-(d) samples in which the coverage layer was produced at the fluence indicated in the label.

(extracted from images such as those in Fig. 2), and the SPR wavelength (as from Fig. 1) as a function of the fluence used to ablate the $\mathrm{Al}_{2} \mathrm{O}_{3}$ target when producing the covering layer. The values included at zero fluence correspond to those obtained for the reference sample. It is seen that while for the lowest studied fluence, $0.3 \mathrm{~F}_{0}$, the covered and uncovered NPs have a similar mean diameter, the latter reduces approximately a factor of 2 for the highest fluence studied $\left(3.3 \mathrm{~F}_{0}\right)$. The metal content $[\mathrm{Au}$ ] follows the same trend, and in fact, diameter and $[\mathrm{Au}]$ are proportional similarly to what has generally been reported. ${ }^{7,8,20}$ The SPR wavelength follows the same trend of diameter and $[\mathrm{Au}]$ up to $\mathrm{F}_{0}$ and is no longer noticeable for higher fluences in spite of the NPs have a mean diameter of $3 \pm 1 \mathrm{~nm}$ for $3.3 \mathrm{~F}_{0}$ (Fig. 2(d)).

Figure 3(b) shows the deposition rate of $\mathrm{Al}_{2} \mathrm{O}_{3}$ during coverage and Fig. 3(c) shows the fraction of sputtered metal $[\mathrm{Au}]_{\%}$ as a function of the fluence used to ablate the $\mathrm{Al}_{2} \mathrm{O}_{3}$ target during coverage. $[\mathrm{Au}]_{\%}$ was calculated from the experimental data measured by RBS as ([Au $\left.]_{\text {ref }}-[\mathrm{Au}]\right) /[\mathrm{Au}]_{\mathrm{ref}}$, where $[\mathrm{Au}]_{\text {ref }}$ is the amount of gold at the surface of the reference sample, i.e., the amount of metal before coverage. It is

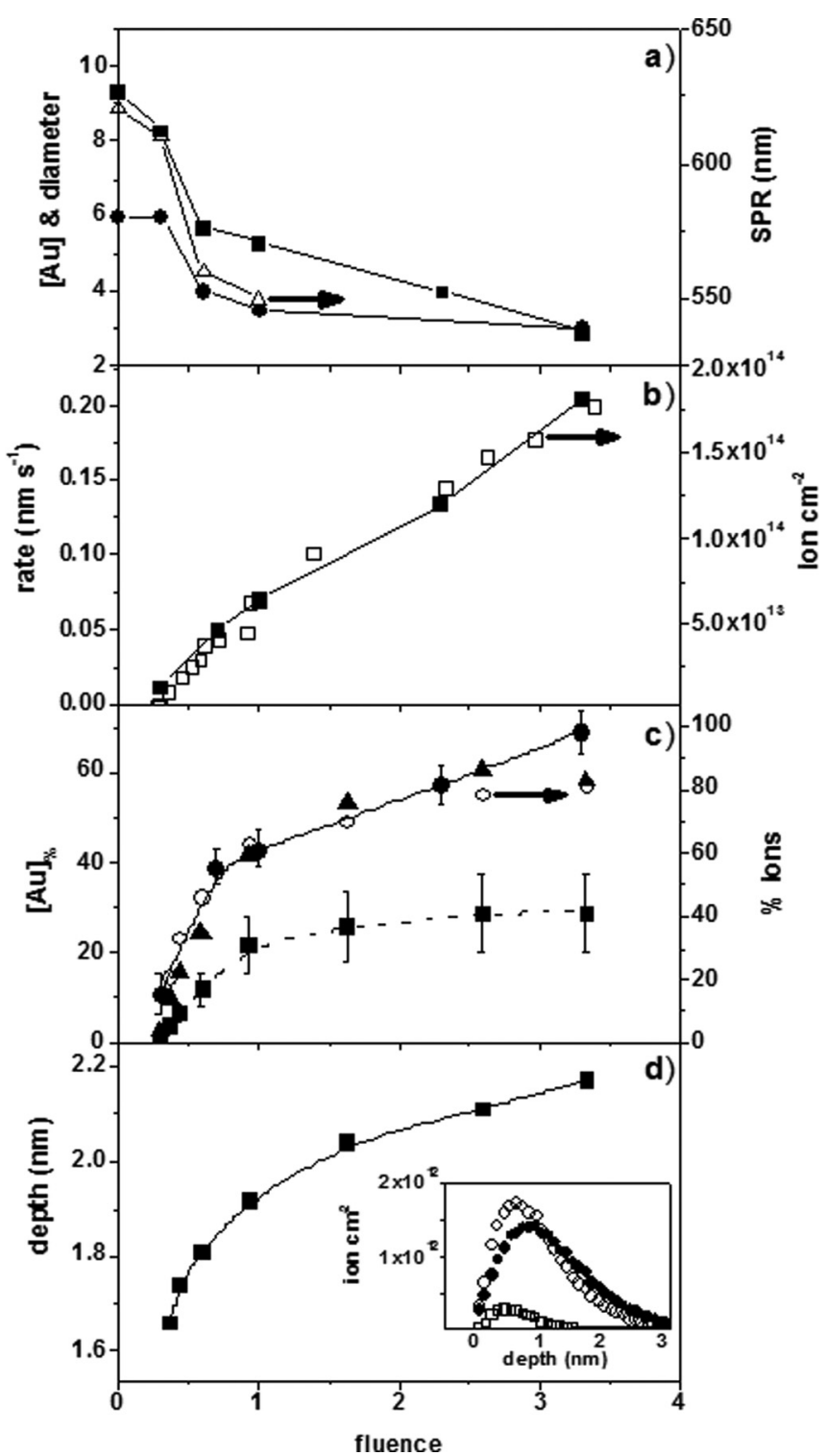

FIG. 3. Evolution of several experimental and calculated parameters as a function of the $\mathrm{X} \mathrm{F}_{0}$ fluence $\left(\mathrm{F}_{0}=2.7 \mathrm{~J} \mathrm{~cm}^{-2}\right)$ to ablate the $\mathrm{Al}_{2} \mathrm{O}_{3}$ target during the production of the covering layer. (a) (ם) Gold content $[\mathrm{Au}]$ in $\times 10^{15}$ atoms $\mathrm{cm}^{-2}$, (-) mean diameter of NPs in nm and $(\triangle)$ SPR wavelength; the values for fluence 0 correspond to the reference sample. (b) (ם) Deposition rate of a- $\mathrm{Al}_{2} \mathrm{O}_{3}$ and $(\square)$ ion yield from Ref. 16. (c) (-) Experimental fraction of sputtered metal $[\mathrm{Au}]_{\%}$ with respect to the reference sample and calculated fraction using the models in Refs. 25 (ם) and $27(\mathbf{\Delta})$; (○) \% of ions arriving to the substrate having $\mathrm{KE}>200 \mathrm{eV}$ from Ref. 16. (d) Effective implantation depth of aluminum ions in Au during the first pulse taking only into account arriving ions with $\mathrm{KE}>200 \mathrm{eV}$. The inset in (d) shows the calculated implanted profile during the first pulse with $(\square) 0.3 \mathrm{~F}_{0}$ and $(\bigcirc, \bullet)$ $0.7 \mathrm{~F}_{0}$ and considering that all ions $(\square, \bigcirc)$ or only those having $\mathrm{KE}>200 \mathrm{eV}$ (-) contribute to the implantation. All lines are guidelines.

seen that while the deposition rate increases monotonously as fluence is increased, the sputtering fraction exhibits a two slope behavior: it increases sharply up to $\approx \mathrm{F}_{0}$ and with a slower slope for higher fluences.

\section{DISCUSSION}

The results obtained in this work clearly show that the fluence used to ablate the target for covering the NPs has important consequences on the final amount of metal and this 
certainly affects the NPs dimensions and optical response. Increasing fluence is well known to increase the amount of ablated material and thus, the deposition rate, consistently to what is shown in Fig. 3(b). In addition, the increase of fluence is known to shift the maximum of the KE distributions $(\mathrm{N}(\mathrm{E}))$ of the species arriving to the substrate towards higher values. ${ }^{21,22}$ However, what is not so well documented is the significant extension of the tail of $\mathrm{N}(\mathrm{E})$ of ions arriving to the substrate to values as high as $1.5 \mathrm{keV}$ as it has recently been reported for the case of ablation of an $\mathrm{Al}_{2} \mathrm{O}_{3}$ target with $193 \mathrm{~nm}$ photons for fluences similar to the highest fluence studied in this work. ${ }^{16}$

Since it was earlier reported that ions having $\mathrm{KE} \geq 200 \mathrm{eV}$ were responsible for sputtering of metal from NPs during their coverage with $\mathrm{Al}_{2} \mathrm{O}_{3}{ }^{7}$ we have, respectively, included in Figs. 3(b) and 3(c) the ion yield and the percentage of these energetic ions as a function of fluence taken from Ref. 16. The comparison of these data to the experimental data obtained in the present work shows that while the deposition rate of coverage follows the monotonous increase of the ion yield, the fraction of sputtered metal atoms follows well the trend of energetic ions, i.e., both magnitudes exhibiting a two-slope behavior with an inflection $\approx \mathrm{F}_{0}$. It is worth to point here that the experimental conditions used for ablating the $\mathrm{Al}_{2} \mathrm{O}_{3}$ target in Refs. 7 and 16 are very similar to those used in this work, in terms of laser wavelength $(193 \mathrm{~nm})$ that is particularly important since the photon energy is higher than the ionization potential of Al. This makes the ionic component of the plasma much higher and energetic than when using longer wavelengths. ${ }^{23}$

The $\mathrm{Al}_{2} \mathrm{O}_{3}$ coverage related effects on Au NPs at a fluence $\mathrm{F}_{0}$ as a function of the initial metal content were earlier simulated using the N(E) reported in Ref. 24 upon ablation of an $\mathrm{Al}$ target. ${ }^{7}$ The calculations were performed in the frame of three different models and it was shown that the Yamamura and Tawara model ${ }^{25}$ provides the best approximation. In this work, we have thus used the latter model for calculating the ion yield using the $\mathrm{N}(\mathrm{E}) \mathrm{s}$ upon ablation of $\mathrm{Al}_{2} \mathrm{O}_{3}$ at $193 \mathrm{~nm}$ that are now available from Ref. 16. We have also taken into account the dependence of the cohesive energy of NPs on their diameter reported elsewhere. ${ }^{7,26}$ Generally, the results show that the amount of sputtered atoms during the first pulse is in the range of $1-10 \times 10^{13}$ atoms $\mathrm{cm}^{-2}$, the higher the fluence the higher the value. In addition, the $3.0 \pm 0.3 \times 10^{13}$ atoms $\mathrm{cm}^{-2}$ value achieved in the present work for $\mathrm{F}_{0}$ and for NPs having initially a diameter of $6 \pm 1 \mathrm{~nm}$ is very similar to the one reported earlier for a mean diameter of $3 \pm 1 \mathrm{~nm}$. $^{7}$ This result supports further the earlier conclusion that the model used leads to SYs that depend little on the NPs dimensions at least for NPs having diameters in the studied range. It is worth pointing out that the model assumes a continuous metal layer and the discontinuous character is taken into account through a diameter dependent cohesive energy and by weighting the results by the experimentally determined metal coverage. ${ }^{7}$

The calculated amount of sputtered atoms during the first pulse follows an approximately linear dependence on fluence rather than the two slope dependence shown by the experimental data in Fig. 3(c). This could be related to the fact that experimental data refer to total amount of sputtered atoms while the calculated ones refer to those sputtered in the first pulse and the amount of sputtered atoms per pulse decreases almost exponentially as the number of pulses increases and becomes negligible for $1 \mathrm{~nm}$ at $\mathrm{F}_{0} .{ }^{7}$ Assuming such an exponential decay with the a- $\mathrm{Al}_{2} \mathrm{O}_{3}$ layer thickness, we have calculated the total amount of sputtered atoms and plotted in Fig. 3(c) their proportion with respect to the reference sample to ease the comparison between experimental and calculated data. The error bars arise from the experimentally measured coverage used to weight the calculated results. It can be seen that the calculated data follow the same trend than the experimental values but their values are approximately half than the experimental ones similarly to what was earlier reported. ${ }^{7}$ We refined the calculations by taking into account that each pulse in the $\mathrm{Al}_{2} \mathrm{O}_{3}$ target sputters a certain amount of metal that makes next pulse species find metal NPs with smaller diameter and thus coverage. Although the experimental dependence of these parameters (diameter (Fig. 3(a)) and coverage as function of $[\mathrm{Au}]$ ) together with the dependence of the cohesive energy on NPs diameter ${ }^{26}$ were considered, the results were almost identical to those plot in Fig. 3(c) without these refinements.

The results show that the Yamamura and Tawara model with the assumption of an exponential decay of the sputtering rate reproduces well the two-slope evolution of the experimentally determined amount of sputtered atoms and the proportion of energetic ions $(\mathrm{KE}>200 \mathrm{eV})$. The importance of these ions is further supported by the calculations because on the one hand, there are no such ions for $0.3 \mathrm{~F}_{0}$ for which the amount of sputtered atoms both experimental and calculated ones are at least one order of magnitude lower than for higher fluences. On the other hand, we have repeated the calculations taking only into account the energetic ions, and the result was identical to that plot in Fig. 3(c) within $\approx 10 \%-15 \%$ for fluences $\geq \mathrm{F}_{0}$.

However, the calculations using the Yamamura and Tawara model always underestimate the SY of NPs, both in this work and earlier reports. ${ }^{7,10,15}$ This model has been developed for flat surfaces rather than for NPs while molecular dynamics simulations have shown an enhancement of the SY of Au NPs under $25 \mathrm{keV}$ Ga bombardment compared to that of bulk with a maximum for NPs of $8 \mathrm{~nm}$ diameter. ${ }^{27}$ Furthermore, the theoretical description of sputtering of $\mathrm{Au}$ NPs by a flux comparable to that in the current work and using $200 \mathrm{keV} \mathrm{Ar}^{+}$and $\mathrm{Xe}^{+}$required the assumption of a size-dependent sputtering coefficient for the Au NPs. ${ }^{15}$ The enhancement of the SY was explained using a model based on work of Sigmund ${ }^{28}$ and assuming the SY is proportional to the damage caused by the incoming ions. ${ }^{15,27} \mathrm{~A}$ three dimensional Gaussian distribution of the ion-induced damage and spherical NPs were considered. The Gaussian distribution has three geometrical parameters, namely, the depth $a$ with respect to the ion impact point of the maximum of the distribution, and the longitudinal and transversal straggling parameters $\alpha$ and $\beta$. In the current work, these parameters were determined as a function of the KE of the incident ions with the SRIM 2013 software. ${ }^{29}$ For each KE, the normalization factor $\Lambda$ (Eq. (3) in Ref. 27) was determined using the results 
achieved with the Yamamura and Tawara model as the best approximation for the SY for a planar surface. The SY of Au NPs for each KE was finally calculated using Eqs. (5) and (6) in Ref. 27 and we have verified that the calculated dependence of the SY as a function of the diameter of the NPs agree very well with those earlier reported. ${ }^{15,27}$ We have finally integrated the SY for NPs of diameter $6 \mathrm{~nm}$ over KE using the $\mathrm{KE}$ distributions reported in Ref. 16 for $\mathrm{Al}$ ions upon laser ablation of $\mathrm{Al}_{2} \mathrm{O}_{3}$ at $193 \mathrm{~nm}$, following the same procedure described above for the calculations within the Yamamura and Tawara model. The proportion of calculated amount of sputtered atoms with respect to the reference sample is also plotted in Fig. 3(c) where it can be seen that the agreement with the experimental values is very good. Thus, our results support further that a size-dependent sputtering coefficient is essential for predicting the experimental SY of NPs.

The changes of the SPR of the NPs follow the same trend of the NPs dimensions and metal content (Fig. 3(a)) and thus must similarly be linked to ion bombardment related effects during coverage. As fluence increases, the optical spectra in Fig. 1 become generally consistent with the smaller dimensions of the NPs in the covered samples with respect to uncovered one, i.e., the intensity at the SPR decreases and its wavelength blue-shifts as diameter decreases. Therefore, the sputtering effects generally compensate the small red-shift expected by the coverage having a higher refractive index even for the lowest fluence studied $\left(0.3 \mathrm{~F}_{0}\right)^{3-5}$

A similar blue-shift and damping have been reported upon irradiation with $100 \mathrm{MeV} \mathrm{Ag}$ ions and related to the decrease in Ag content due to sputtering. ${ }^{12}$ Also, similar blue-shift and damping of the SPR was reported for the coverage of $\approx 5 \mathrm{~nm}$ diameter $\mathrm{Cu}$ NPs with a- $\mathrm{Al}_{2} \mathrm{O}_{3}$ by PLD for fluences between $0.7 \mathrm{~F}_{0}$ and $1.3 \mathrm{~F}_{0}$ under experimental conditions almost identical to the present work. ${ }^{6}$ The damping of the SPR was discussed in terms of mixing of the species arriving to the substrate during the coverage process with the metal ones and leading to a metal-Al oxide shell that reduces the actual diameter of the metallic core contributing to the SPR. Once it becomes smaller than a critical value, there are no enough electrons contributing to the collective oscillations and the SPR is no longer distinguishable.

However, this reasoning does not explain why the SPR is no longer noticeable for fluences $>F_{0}$ in spite of the samples contain NPs of $3 \pm 1 \mathrm{~nm}$ as shown in Fig. 2(d) and uncovered NPs of that size have been reported to exhibit an SPR around $575 \mathrm{~nm}^{7}$ We have used the SRIM 2013 software $^{29}$ for calculating the implantation range of $\mathrm{Al}$ in $\mathrm{Au}$ during the first pulse as a function of fluence. ${ }^{16}$ The inset in Fig. 3(d) shows the resulting implantation profiles normalized to the ion yield plot in Fig. 3(b) for $0.3 \mathrm{~F}_{0}$ and $0.7 \mathrm{~F}_{0}$. They show that while implantation effects are negligible for depths higher than $1 \mathrm{~nm}$ for the lowest fluence $\left(0.3 \mathrm{~F}_{0}\right)$, they become relevant up to a depth of $3 \mathrm{~nm}$ for $0.7 \mathrm{~F}_{0}$. In addition, the profile produced taking only into account ions having $\mathrm{KE}>200 \mathrm{eV}$ for $0.7 \mathrm{~F}_{0}$ is also included. It has similar shape and extension than that taking into account ions having any $\mathrm{KE}$, thus evidencing that the implantation effects are mainly caused by these energetic ions as suggested earlier. ${ }^{7}$
Furthermore, as fluence increases (not shown), the profiles achieved considering all ions or only the energetic ones become even closer. These profiles have been calculated for the first pulse that is the one producing the highest depth since next pulses will find that the metal is partially covered by $\mathrm{Al}_{2} \mathrm{O}_{3}$ and thus will produce similar profiles but shifted to lower depths. We have defined the effective implantation depth as the highest depth of the FWHM of the implantation distribution obtained in the first pulse considering only the energetic ions. Figure 3(d) shows that such implantation depth varies in the range of $\approx 1.6-2.2 \mathrm{~nm}$ as fluence increases. However, the profiles extend deeper than $3 \mathrm{~nm}$ as seen in the inset for $0.7 \mathrm{~F}_{0}$. For this fluence, $\approx 3 \%$ of the energetic species implant deeper than $3 \mathrm{~nm}$ with the first pulse and this proportion increases up to $\approx 10 \%$ for the highest fluence. Furthermore, the depths follow a two-slope behavior with the change around $\mathrm{F}_{0}$, similarly to what happens with the NPs diameter and metal content (Fig. 3(a)) or the sputtering rate (Fig. 3(c)). Figures 1 and 3(a) show that a SPR is still resolvable for a covered sample having NPs with an in-plane average diameter of $3.5 \pm 0.5 \mathrm{~nm}$ that was produced using a fluence $\mathrm{F}_{0}$ for the coverage but is not resolvable for higher fluences leading to NPs having slightly smaller diameters. Therefore, we can conclude that the coverage of the NPs produces implantation of aluminum ions having $\mathrm{KE}>200 \mathrm{eV}$. When the effective implantation depth extends along most of the diameter of the NPs, the mixture formed exhibits no longer a plasmonic response in the visible part of the spectrum.

Finally, while the mixing process has little relevance when working at low fluences (compare spectra of reference sample and that prepared at $0.3 \mathrm{~F}_{0}$ in Fig. 1), the sputtering process is not negligible $(<10 \pm 5 \%)$ for a laser fluence that is close to the ion threshold upon ablation of $\mathrm{Al}_{2} \mathrm{O}_{3}, \sim 0.3$ $\mathrm{F}_{0},{ }^{16}$ i.e., under a quasi-thermal plasma expansion regime for which there are no ions with $\mathrm{KE}>200 \mathrm{eV}$. Thus, the sputtering process cannot be completely avoided but only limited during the coverage of NPs with $\mathrm{Al}_{2} \mathrm{O}_{3}$ using $193 \mathrm{~nm}$ photons. Since both the high degree of ionization and the high $\mathrm{KE}$ of ions in the $\mathrm{Al}_{2} \mathrm{O}_{3}$ plasma produced upon ablation at $193 \mathrm{~nm}$ are due to the photon energy being higher than the photoionization potential of $\mathrm{Al}$, one possible way to reduce further the sputtering processes is to work at longer wavelengths $(\geq 248 \mathrm{~nm})$ for which KEs are much lower as recently reported. ${ }^{23}$

\section{CONCLUSIONS}

The covering process has important consequences on the optical properties of embedded NPs that are related to changes in the morphological and compositional features of the NPs. These changes are linked to the kinetic energy of the species bombarding the metal NPs during their coverage, particularly ions having $\mathrm{KE}>200 \mathrm{eV}$. The effect of this bombardment is twofold. On the one hand, it sputters a significant amount of metal that is not negligible for fluences close to the ion threshold, the higher the fluence the higher the sputtered fraction (up to 70\%). On the other hand, it is responsible for implantation of the coverage species in the 
volume of the NPs up to depths $>3 \mathrm{~nm}$, leading to a change of composition. Generally, the optical response is consistent with the decrease in the overall dimensions of the NPs produced by sputtering and when the volume modified by implantation becomes comparable to that of the NP, the SPR associated to Au NPs is no longer noticeable. Finally, standard models for ion bombardment are enough to predict the trend and order of magnitude of the SY but lead to values that are at least $\approx 2$ times smaller than the experimental ones. The experimental values are well fitted by combining the standard models with a size-dependent approach for the SY.

\section{ACKNOWLEDGMENTS}

This work has been partially supported by MAT201128345-C02-02 (Spain) project. R.P. acknowledges the grant JCI-2012_13034 from the Juan de la Cierva program. The authors would like to thank J. García from Centro Nacional de Aceleradores, Universidad de Sevilla and CSIC (Spain) for RBS measurements and E. Piscopiello from ENEA, Unità Tecnica Tecnologie dei Materiali Brindisi (UTTMATB) for TEM images.

${ }^{1}$ S. Link and M. A. El-Sayed, Int. Rev. Phys. Chem. 19, 409 (2000), and references therein.

${ }^{2}$ U. Kreibig and M. Vollmer, Optical Properties of Metal Clusters (Springer-Verlag, 1994).

${ }^{3}$ C. F. Bohren and D. R. Huffman, Absorption and Scattering of Light by Small Particles (Wiley, New York, 1983).

${ }^{4}$ G. Xu, M. Tazawa, P. Jin, S. Nakao, and K. Yoshimura, Appl. Phys. Lett. 82, 3811 (2003).

${ }^{5}$ V. Antad, L. Simonot, D. Babonneau, S. Camelio, F. Pailloux, and P. Guérin, J. Nanophotonics 6, 061502 (2012).

${ }^{6}$ R. Serna, A. Suarez-García, C. N. Afonso, and D. Babonneau, Nanotechnology 17, 4588 (2006).

${ }^{7}$ V. Resta, J. Gonzalo, C. N. Afonso, E. Piscopiello, and J. García, J. Appl. Phys. 109, 94302 (2011).
${ }^{8}$ J. Gonzalo, A. Perea, D. Babonneau, C. N. Afonso, N. Beer, J.-P. Barnes, A. K. Petford-Long, D. E. Hole, and P. D. Townsend, Phys. Rev. B 71, 125420 (2005).

${ }^{9}$ Ion Beam Modifications of Surfaces, edited by O. Auciello and R. Kelly (Elsevier, Amsterdam, 1984).

${ }^{10}$ A. Gupta and D. Avasthi, Phys. Rev. B 64, 155407 (2001).

${ }^{11}$ Z. Pásztia, G. Pető, Z. Horváth, O. Geszti, A. Karacs, and L. Gucz, Nucl. Instrum. Methods Phys. Res., Sect. B 178, 131-134 (2001).

${ }^{12}$ U. B. Singh, D. C. Agarwal, S. A. Khan, S. Mohapatra, A. Tripathi, and D. K. Avasthi, J. Phys. D: Appl. Phys. 45, 445304 (2012).

${ }^{13}$ X. Zhou, Q. Wei, K. Sun, and L. Wang, Appl. Phys. Lett. 94, 133107 (2009).

${ }^{14}$ J. Prakash, A. Tripathi, V. Rigato, J. C. Pivin, J. Tripathi, K. H. Chae, S. Gautam, P. Kumar, K. Asokan, and D. K. Avasthi, J. Phys. D: Appl. Phys. 44, 125302 (2011)

${ }^{15}$ A. Klimmer, P. Ziemann, J. Biskupek, U. Kaiser, and M. Flesch, Phys. Rev. B 79, 155427 (2009).

${ }^{16}$ G. Baraldi, A. Perea, and C. N. Afonso, Appl. Phys. A: Mater. Sci. Process. 105, 75 (2011).

${ }^{17}$ A. Castelo, C. N. Afonso, E. Pesce, and E. Piscopiello, Nanotechnology 23, 105603 (2012).

${ }^{18}$ W. Rasband, National Institutes of Health (USA), http://rsbweb.nih.gov/ij/.

${ }^{19}$ V. Resta, A. P. Caricato, A. Loiudice, A. Rizzo, G. Gigli, A. Taurino, M. Catalano, and M. Martino, J. Nanopart. Res. 15, 2017 (2013).

${ }^{20}$ V. Resta, C. N. Afonso, E. Piscopiello, and G. Van Tendeloo, Phys. Rev. B 79, 235409 (2009).

${ }^{21}$ S. Fähler and H.-U. Krebs, Appl. Surf. Sci. 96-98, 61-65 (1996).

${ }^{22}$ B. Toftmann and J. Schou, Appl. Phys. A: Mater. Sci. Process. 112, 197-202 (2013).

${ }^{23}$ R. J. Peláez, C. N. Afonso, M. Bator, and T. Lippert, J. Appl. Phys. 113, 223301 (2013).

${ }^{24}$ F. Claeyssens, S. J. Henley, and M. N. R. Ashfold, J. Appl. Phys. 94(4), 2203 (2003).

${ }^{25}$ Y. Yamamura and H. Tawara, At. Data Nucl. Data Tables 62, 149-253 (1996).

${ }^{26}$ D. Xie, M. P. Wang, and W. H. Qi, J. Phys.: Condens. Matter 16, L401 (2004).

${ }^{27}$ T. Järvi, J. A. Pakarinen, A. Kuronen, and K. Nordlund, EPL 82, 26002 (2008).

${ }^{28} \mathrm{P}$. Sigmund, "Sputtering by particle bombardment I," in Sputtering by Ion Bombardment: Theoretical Concepts, Top. Appl. Phys., Vol. 47, edited by R. Behrisch (Springer-Verlag, 1981), Chap. 2, pp. 9-71.

${ }^{29}$ J. F. Ziegler, SRIM Software ver2013.00, http://www.srim.org/. 